\title{
The transfer paradox in welfare space*
}

\author{
Thomas Demuynck ${ }^{\dagger} \quad$ Bram De Rock $^{\ddagger} \quad$ Victor Ginsburgh ${ }^{\S}$
}

February 4, 2015

\begin{abstract}
The transfer paradox describes a situation in which a transfer of endowments between two agents results in a welfare decrease for the recipient and a welfare increase for the donor. It is known that in a two-agent regular exchange economy with an arbitrary number of goods, the transfer paradox occurs only if the price equilibrium is unstable. In this paper, we show that in the space of welfare weights, the set of stable equilibria and the set of no-transfer paradox equilibria coincide. As a corollary we also obtain that for two agents and an arbitrary number of goods, the index of an equilibrium in price space coincides with its index in welfare space.
\end{abstract}

JEL codes: D51, D60

Keywords: welfare equilibrium, exchange economy, transfer paradox

\section{Introduction}

In this paper, we show that in a two agent economy with an arbitrary number of goods, the transfer paradox occurs if and only if the equilibrium is unstable

\footnotetext{
${ }^{*}$ We thank Yves Balasko for useful comments.

${ }^{\dagger}$ Maastricht University, Tongersestraat 53, 6370 Maastricht, Netherlands. Email: t.demuynck@maastrichtuniversity.nl.

${ }_{\ddagger}^{\ddagger}$ ECARES, Université Libre de Bruxelles. Avenue F. D. Roosevelt 50, CP 114, B-1050 Brussels, Belgium. E-mail: bderock@ulb.ac.be. Bram De Rock gratefully acknowledges the European Research Council (ERC) for his Starting Grant 263707.

${ }^{\S}$ ECARES, Université Libre de Bruxelles. Avenue F. D. Roosevelt 50, CP 114, B-1050 Brussels, Belgium. E-mail: vginsburg@ulb.ac.be.
} 
in welfare space. In this introduction, we position our paper in the literature and discuss the intuition behind the mechanics of the transfer paradox in welfare space.

The transfer paradox. The transfer paradox takes place in a two agent exchange economy, when a transfer from the first agent to the second leads to a gain of welfare for the donor and a loss in welfare for the recipient. The mechanism underlying the transfer paradox is well known. Given that a redistribution of resources not only shifts the endowments, but also the equilibrium prices, it is possible that the positive income effect for the donor is outweighed by a negative price effect, and therefore makes her worse off.

The existence of the transfer paradox was first noticed by Leontief (1947). Shortly afterwards, Samuelson $(1952,1954)$ demonstrated (in a two agent and two commodity exchange economy) that a transfer paradox can only occur if the initial equilibrium is tâtonnement unstable. ${ }^{1}$ Since then, many variations of the transfer paradox have been studied. The focus was particular on the use of specific preferences and the impact of variations in the number of goods and the number of agents; see for instance Gale (1974), Balasko (1975), Chichilnisky (1980) and Bhagwati, Brecher, and Hatta (1983), Geanakoplos and Heal (1983), Srinivasan and Bhagwati (1983), Chichilnisky (1983), Polemarchakis (1983) and Safra (1984). ${ }^{2}$

Recently, Balasko (2014) provided the first complete characterization of the transfer paradox. He showed that in a two agent exchange model, with an arbitrary number of commodities and arbitrary (well-behaved) preferences, the transfer paradox can occur at a regular equilibrium if and only if the equilibrium has an index value of -1 . Given that equilibria with index value -1 are tâtonnement unstable (the reverse is not always true if the number of goods is larger than two) this shows that the transfer paradox cannot occur at a stable equilibrium.

Equilibria in price and welfare space. The usual procedure to find equilibria in an exchange economy is by first deriving the offer curves for the agents in the economy, i.e. the individual excess demands as a function

\footnotetext{
${ }^{1}$ See also Mundell (1960), Balasko (1978), Postlewaite and Webb (1984).

${ }^{2}$ There were also many other extensions focusing on whether offer curves are elastic (Majumdar and Mitra (1985), Yano (1983), Dixit (1983)), whether goods are substitutes or complements (Majumdar and Mitra (1985)), etc..
} 
of prices and individual endowments. Next, the equilibrium price vector is computed as the price for which the aggregate excess demand is zero for all goods simultaneously. A Walrasian equilibrium is said to be tâtonnement stable if small deviations from the equilibrium allocation converge back to the equilibrium, when prices are raised for goods with excess demand and lowered for goods with excess supply. This describes a dynamic process where demands are instantaneously adjusted to balance the individual budgets and prices adjust 'more slowly' in response to the excess demand or supply on each market.

An alternative approach to compute equilibria in an exchange equilibrium, due to Negishi (1960), is to first retrieve all allocation on the contract curve together with the marginal rates of substitution (i.e. the shadow prices). Subsequently, one needs to compute the welfare weights attached to the agents in the economy (i.e. allocation on the contract curve) for which the budget constraints of all individuals are simultaneously binding (given the corresponding shadow prices). In order to make a distinction with the approach above, we call this an equilibrium in welfare space. By construction, each equilibrium in welfare space corresponds to a unique Walrasian quilibrium (and vice versa). See also Section 2 for more discussion.

Similarly to tâtonnement stability, it is also possible to discuss the stability of the equilibria in welfare space. The dynamics are not determined by price variations, but by variation in the welfare weights, which we denote by $\alpha$. In this way, it is possible to distinguish between equilibria that are $\alpha$-stable or $\alpha$-unstable. The difference with the tâtonnement adjustment process is that for $\alpha$-stability, individual demands are immediately adjusted in order to clear the markets, while welfare weights (and the corresponding shadow prices) change 'more slowly' in response to individual excess budgets or deficits. That is, in $\alpha$-stable equilibria, welfare weights are increased for agents with a budget surplus and are decreased for agents with a budget deficit.

Our contribution. In this paper we provide a characterization of the transfer paradox in terms of $\alpha$-stability of an equilibrium. As such, we complement the existing literature, briefly described above, which mainly focuses on Walrasian equilibria. The main result of this paper is that the transfer paradox takes place if and only if the equilibrium is $\alpha$-unstable. The intuition for this result is as follows: a transfer of endowments from one agent to 
another creates a budget surplus for the receiving agent. If the equilibrium is $\alpha$-stable, then this surplus leads to an increase of the welfare weight for this agent. This increase of the welfare weight, in turn, increases the equilibrium utility level of the recipient. On the other hand, if the equilibrium is $\alpha$-unstable, then the budget surplus of the receiving agent (due to the transfer) decreases her welfare weight and, consequently, her equilibrium utility level.

Using Balasko (2014)'s result, we obtain as a corollary of our characterisation that in a two agent economy, the Walrasian equilibrium has index -1 if and only if the corresponding equilibrium in welfare space is $\alpha$-unstable. Or, given that in a two agent economy $\alpha$-stability coincides with an index of +1 in welfare space, we obtain that the index in welfare space coincides with the index in $p$-space. This conclusion provides further insight for why the transfer paradox does not always occur at tâtonnement unstable equilibria.

The rest of the paper unfolds as follows. The next section discusses the concept of a welfare equilibrium and $\alpha$-stability in a two agent exchange economy with an arbitrary number of goods. Section 3 presents our main result. Section 4 concludes.

\section{Welfare equilibria}

We briefly present the notions of an equilibrium in welfare space and the link to the corresponding Walrasian equilibrium. See also Ginsburgh and Waelbroeck (1979) and Mantel (1971) for more details.

We consider an exchange economy with $n$ goods and two agents. We denote the two agents by $A$ and $B$ and endow them with strictly monotone, strictly concave and $C^{1}$ utility functions $u^{A}$ and $u^{B}$. Individual $A$ consumes the bundle $\mathbf{q}^{A} \in \mathbb{R}_{+}^{n}$ and $B$ consumes the bundle $\mathbf{q}^{B} \in \mathbb{R}_{+}^{n}$. Endowments of the individuals are given by $\boldsymbol{\omega}^{A} \in \mathbb{R}_{+}^{n}$ and $\boldsymbol{\omega}^{B} \in \mathbb{R}_{+}^{n}$. Let $\boldsymbol{\omega}=\boldsymbol{\omega}^{A}+\boldsymbol{\omega}^{B}$ be the aggregate endowment in the economy.

A price (row) vector $\mathbf{p}^{*} \in \mathbb{S}^{n}$, where $\mathbb{S}^{n}$ is the unit simplex, is a Walrasian equilibrium price vector if,

$$
\mathbf{q}^{A}\left(\mathbf{p}^{*}, \mathbf{p}^{*} \boldsymbol{\omega}^{A}\right)+\mathbf{q}^{B}\left(\mathbf{p}^{*}, \mathbf{p}^{*} \boldsymbol{\omega}^{B}\right)-\boldsymbol{\omega}=\mathbf{0},
$$


with

$$
\begin{aligned}
& \mathbf{q}^{A}\left(\mathbf{p}^{*}, \mathbf{p}^{*} \boldsymbol{\omega}^{A}\right)=\arg \max _{\mathbf{q}} u^{A}(\mathbf{q}) \text { s.t. } \mathbf{p}^{*} \mathbf{q}^{A}=\mathbf{p}^{*} \boldsymbol{\omega}^{A} \text { and, } \\
& \mathbf{q}^{B}\left(\mathbf{p}^{*}, \mathbf{p}^{*} \boldsymbol{\omega}^{B}\right)=\arg \max _{\mathbf{q}} u^{B}(\mathbf{q}) \text { s.t. } \mathbf{p}^{*} \mathbf{q}^{B}=\mathbf{p}^{*} \boldsymbol{\omega}^{B} .
\end{aligned}
$$

At the equilibrium, both agents choose the optimal bundle subject to their budget constraint, and equilibrium prices are determined by the fact that all markets are simultaneously cleared (i.e. the excess demand functions are zero).

An alternative way to determine the equilibrium allocation is by using the dual approach of Negishi (1960). This approach departs from the following welfare maximization program,

$$
\max _{\mathbf{q}^{A}, \mathbf{q}^{B}} \alpha u_{A}\left(\mathbf{q}^{A}\right)+u_{B}\left(\mathbf{q}^{B}\right) \text { s.t. } \mathbf{q}^{A}+\mathbf{q}^{B}=\boldsymbol{\omega},
$$

where $\alpha \geq 0$ is a parameter that determines the welfare weight given to $A$ (relative to $B$ ). This maximization problem considers the bundles $\mathbf{q}^{A}$ and $\mathbf{q}^{B}$ that maximize a weighted sum of utilities subject to the total resource constraint.

Let $\mathbf{q}^{A}(\alpha, \boldsymbol{\omega})$ and $\mathbf{q}^{B}(\alpha, \boldsymbol{\omega})$ be the interior solution of this welfare maximization problem. ${ }^{3}$ Then, by varying $\alpha$ over $\mathbb{R}_{+}$these bundles trace out the entire contract curve (i.e. set of Pareto optimal allocations). Next, let $\boldsymbol{\lambda}(\alpha, \boldsymbol{\omega})$ represent the vector of Lagrange multipliers of the welfare maximization problem. From the first order conditions, we obtain that at an interior solution, for any two goods $i$ and $j$,

$$
\frac{\frac{\partial u^{A}\left(\mathbf{q}^{A}(\alpha, \boldsymbol{\omega})\right)}{q_{i}^{A}}}{\frac{\partial u^{A}\left(\mathbf{q}^{A}(\alpha, \boldsymbol{\omega})\right)}{q_{j}^{A}}}=\frac{\lambda_{i}(\alpha, \boldsymbol{\omega})}{\lambda_{j}(\alpha, \boldsymbol{\omega})}=\frac{\frac{\partial u^{B}\left(\mathbf{q}^{B}(\alpha, \boldsymbol{\omega})\right)}{q_{i}^{B}}}{\frac{\partial u^{B}\left(\mathbf{q}^{B}(\alpha, \boldsymbol{\omega})\right)}{q_{j}^{B}}} .
$$

As such, we see that the vector $\boldsymbol{\lambda}(\alpha, \boldsymbol{\omega})$ corresponds to the shadow prices of the agents at the allocation $\left(\mathbf{q}^{A}(\alpha, \boldsymbol{\omega}), \mathbf{q}^{B}(\alpha, \boldsymbol{\omega})\right)$.

The welfare weight $\alpha^{*}$ is said to be an welfare equilibrium if

$$
\boldsymbol{\lambda}\left(\alpha^{*}, \boldsymbol{\omega}\right) \cdot\left(\boldsymbol{\omega}^{A}-\mathbf{q}^{A}\left(\alpha^{*}, \boldsymbol{\omega}\right)\right)=0 .
$$

\footnotetext{
${ }^{3}$ For simplicity, we will only focus to settings where the solutions are interior.
} 
Note that the vector $\boldsymbol{\lambda}\left(\alpha^{*}, \boldsymbol{\omega}\right)$ corresponds to the equilibrium price vector, which provides a formal link between our two types of equilibria. ${ }^{4}$ The above condition requires that at the equilibrium, the total expenditure of agent $A$ should equal the total worth of her endowment. Using the resource constraint and the fact that $\boldsymbol{\omega}^{B}=\boldsymbol{\omega}-\boldsymbol{\omega}^{A}$, we immediately obtain that at an equilibrium, the excess budget should also be equal to zero.

$$
\boldsymbol{\lambda}\left(\alpha^{*}, \boldsymbol{\omega}\right) \cdot\left(\boldsymbol{\omega}^{B}-\mathbf{q}^{B}\left(\alpha^{*}, \boldsymbol{\omega}\right)\right)=0 .
$$

Finally, let us define the excess budget function for $A$,

$$
b\left(\alpha, \boldsymbol{\omega}^{A}, \boldsymbol{\omega}\right)=\boldsymbol{\lambda}(\alpha, \boldsymbol{\omega}) \cdot\left(\boldsymbol{\omega}^{A}-\mathbf{q}_{A}(\alpha, \boldsymbol{\omega})\right) .
$$

The function $b\left(\alpha, \boldsymbol{\omega}^{A}, \boldsymbol{\omega}\right)$ is larger than zero if the worth of $A$ 's endowments is larger than the worth of her consumption evaluated at the shadow prices. Then, $\alpha^{*}$ constitutes a welfare equilibrium if and only if $b\left(\alpha^{*}, \boldsymbol{\omega}^{A}, \boldsymbol{\omega}\right)=0$. This equilibrium is said to be $\alpha$-stable if

$$
\frac{\partial b\left(\alpha^{*}, \boldsymbol{\omega}^{A}, \boldsymbol{\omega}\right)}{\partial \alpha}<0
$$

The intuition is that local deviations from the equilibrium (by a change in $\alpha$ from its equilibrium value) converge back to the equilibrium, by increasing $\alpha$ if agent $\mathrm{A}$ has a budget surplus and decreasing $\alpha$ in cases of a budget deficit. In this two-agent setting, $\alpha$-stable equilibria have index +1 in welfare space and $\alpha$-unstable equilibria have index -1 .

It is important to note that, although there is a formal link between the two types of equilibria, the (un)stability of the Walrasian equilibrium should not always coincide with the (un)stability of the corresponding welfare equilibrium. Indeed, as soon as there are more than two goods, it is possible that there are equilbria that are $p$-unstable and $\alpha$-stable. For a more indepth discussion about the relation between the two tâtonnement processes, we refer to Mantel (1971) and Ginsburgh and Waelbroeck (1979). Below, we will link $\alpha$-stability to the occurrence of a transfer paradox.

\footnotetext{
${ }^{4}$ For completeness, the ratio of the two Lagrange multipliers of the maximization problems in the Walrasian equilibrium is equal to the welfare weight. This provides the formal link in the opposite direction.
} 


\section{$3 \alpha$-stability and the transfer paradox}

Applying the envelope theorem to the function,

$$
W(\alpha, \boldsymbol{\omega})=\max _{\mathbf{q}^{A}, \mathbf{q}^{B}} \alpha u^{A}\left(\mathbf{q}^{A}\right)+u^{B}\left(\mathbf{q}^{B}\right) \text { s.t. } \mathbf{q}^{A}+\mathbf{q}^{B}=\boldsymbol{\omega}
$$

gives

$$
\frac{\mathrm{d} W(\alpha, \boldsymbol{\omega})}{\mathrm{d} \alpha}=u^{A}\left(\mathbf{q}^{A}(\alpha, \boldsymbol{\omega})\right)
$$

To simplify the notation, let us denote $v^{A}(\alpha, \boldsymbol{\omega}) \equiv u^{A}\left(\mathbf{q}^{A}(\alpha, \boldsymbol{\omega})\right)$. By strict convexity of $W(\alpha, \boldsymbol{\omega})$, see Lemma 2 in the Appendix, we have

$$
\frac{\mathrm{d}^{2} W(\alpha, \boldsymbol{\omega})}{\mathrm{d} \alpha^{2}}=\frac{\partial v^{A}(\alpha, \boldsymbol{\omega})}{\partial \alpha}>0 .
$$

Let $\omega_{j}^{B}>0$ and consider the following parametrization of the endowments,

$$
\begin{aligned}
& \boldsymbol{\omega}^{A}(t)=\left(\begin{array}{llllll}
\omega_{1}^{A} & \omega_{2}^{A} & \ldots & \omega_{j}^{A}+t & \ldots & \omega_{r}^{A}
\end{array}\right) . \\
& \boldsymbol{\omega}_{B}(t)=\left(\begin{array}{llllll}
\omega_{1}^{B} & \omega_{2}^{B} & \ldots & \omega_{j}^{B}-t & \ldots & \omega_{r}^{B}
\end{array}\right) .
\end{aligned}
$$

By increasing $t$, we increase the transfer of endowment $j$ from $B$ to $A$. The particular endowment, or combination of endowments that is transferred is not really important for our reasoning below. As such the above chosen parametrization is without loss of generality. Finally, notice that for all values of $t, \boldsymbol{\omega}^{A}(t)+\boldsymbol{\omega}^{B}(t)=\boldsymbol{\omega}$. In other words, aggregate endowments are independent of $t$.

For each value of $t$ in a neighbourhood around 0 , consider the welfare weight $\alpha^{*}(t)$ associated to a regular equilibrium. Balasko (1992) showed that the set of regular equilibria is an open dense subset of the equilibrium manifold, so this restriction to regular equilibria is minor. We further assume that $\alpha^{*}(t)$ is differentiable in $t$. Then we have that the welfare effects on $A$ of a small increase in the transfer from $B$ to $A$ (evaluated at $t=0$ ) is given by,

$$
\left.\frac{d u^{A}}{d t}\right|_{t=0}=\frac{\partial v^{A}\left(\alpha^{*}(0), \boldsymbol{\omega}\right)}{\partial \alpha} \frac{d \alpha^{*}(0)}{d t}
$$


We say that the transfer paradox occurs if $A$ loses (and consequently, by Pareto optimality, $B$ gains) despite the increase in transfer from $B$ to $A$; i.e.,

$$
\left.\frac{d u^{A}}{d t}\right|_{t=0}<0 .
$$

Proposition 1. If the solution to the welfare maximization program is interior, then a transfer paradox occurs if and only if the welfare equilibrium is $\alpha$-unstable.

Proof. We know that a transfer paradox occurs if and only if

$$
\frac{\partial v^{A}\left(\alpha^{*}(0), \boldsymbol{\omega}\right)}{\partial \alpha} \frac{d \alpha^{*}(0)}{d t}<0
$$

From equation (1) we know that the first term is strictly positive. As such, the transfer paradox occurs if and only if

$$
\frac{d \alpha^{*}(0)}{d t}<0
$$

Now, consider the excess budget function

$$
b\left(\alpha^{*}(t), \boldsymbol{\omega}^{A}(t), \boldsymbol{\omega}\right)=\boldsymbol{\lambda}\left(\alpha^{*}(t), \boldsymbol{\omega}\right) \cdot\left(\boldsymbol{\omega}^{A}(t)-\mathbf{q}^{A}\left(\alpha^{*}(t), \boldsymbol{\omega}\right)\right) .
$$

The equilibrium constraint requires that $b\left(\alpha^{*}(t), \boldsymbol{\omega}^{A}(t), \boldsymbol{\omega}\right)=0$ for all $t$. As such, differentiating the equilibrium condition with respect to $t$, and evaluating at $t=0$ gives,

$$
\begin{aligned}
& 0=\left.\frac{\mathrm{d} b}{\mathrm{~d} t}\right|_{t=0}=\frac{\partial b\left(\alpha^{*}(0), \boldsymbol{\omega}^{A}(0), \boldsymbol{\omega}\right)}{\partial \alpha} \frac{d \alpha^{*}(0)}{d t}+\frac{\partial b\left(\alpha^{*}(0), \boldsymbol{\omega}^{A}(0), \boldsymbol{\omega}\right)}{\partial \omega_{j}^{A}} \\
\Longleftrightarrow & 0=\frac{\partial b\left(\alpha^{*}(0), \boldsymbol{\omega}^{A}(0), \boldsymbol{\omega}\right)}{\partial \alpha} \frac{d \alpha^{*}(0)}{d t}+\lambda^{j}\left(\alpha^{*}(0), \boldsymbol{\omega}\right) \\
\Longleftrightarrow & \frac{\partial b\left(\alpha^{*}(0), \boldsymbol{\omega}^{A}(0), \boldsymbol{\omega}\right)}{\partial \alpha} \frac{d \alpha^{*}(0)}{d t}=-\lambda^{j}\left(\alpha^{*}(0), \boldsymbol{\omega}\right) .
\end{aligned}
$$

The right hand side of this equality is negative because the Lagrange multipliers (prices) are strictly positive. If the equilibrium is $\alpha$-stable, then, by definition, we have that the first factor on the left hand side, $\frac{\partial b\left(\alpha^{*}, \boldsymbol{\omega}^{A}, \boldsymbol{\omega}\right)}{\partial \alpha}$, is strictly negative. This means that $\frac{d \alpha^{*}(0)}{d t}>0$ and thus implies that the transfer paradox does not occur.

On the other hand, if the equilibrium is not stable, then $\frac{\partial b\left(\alpha^{*}, \boldsymbol{\omega}^{A}, \boldsymbol{\omega}\right)}{\partial \alpha}>$ 0 , which means that $\frac{d \alpha^{*}(0)}{d t}<0$ and, consequentially, the transfer paradox occurs. 


\section{Conclusion}

We showed that in a two agent exchange economy with an arbitrary number of goods, a transfer paradox occurs (at a regular equilibrium) if and only if the welfare equilibrium is $\alpha$-unstable. This result complements the existing literature, which mainly focuses on Walrasian equilibria.

Our result provides an alternative, and complimentary, explanation for the relation between tâtonnement stability and the transfer paradox. That is, Balasko (2014) showed that in a two agent exchange model, with an arbitrary number of commodities, the transfer paradox at a Walrasian equilibrium if and only if the equilibrium has an index value of -1 . Given that the Walrasian equilibria with index value -1 are tâtonnement unstable, this demonstrates that a transfer paradox cannot occur at a stable equilibrium. The opposite is however not true, which explains why the transfer paradox does not always occur at unstable Walrasian equilibria.

Using Balasko's result, we obtain as a corollary of our characterisation that in a two agent economy, the Walrasian equilibrium has index -1 if and only if it the corresponding welfare equilibrium is $\alpha$-unstable. Or, given that in a two agent economy $\alpha$-stability coincides with an index of +1 in welfare space, we obtain that the index in welfare space coincides with the index in $p$-space. As such we have shown that there can be equilibria that are tâtonnement unstable, but at the same time $\alpha$-stable. It is only at these type of tâtonnement unstable equilibria that the transfer paradox does not occur.

For simplicity, and comparability to Balasko (2014), we restricted ourselves to a setting with two agents. Given that there are unstable Walrasian equilibria with an index of +1 , it would be interesting to explore if a similar result is true in welfare space. In other words, do there exist, in a setting with more than two agents, unstable welfare equilibria at which the transfer paradox does not occur? We leave this for future research.

\section{A Proofs of the lemmata}

Lemma 1. For interior solutions solutions of the welfare maximization program, if $\alpha \neq \alpha^{\prime}$ then $\mathbf{q}^{A}(\alpha, \boldsymbol{\omega}) \neq \mathbf{q}^{A}\left(\alpha^{\prime}, \boldsymbol{\omega}\right)$.

Proof. Let us prove this ad absurdum and assume that $\mathbf{q}^{A}(\alpha, \boldsymbol{\omega})=\mathbf{q}^{A}\left(\alpha^{\prime}, \boldsymbol{\omega}\right)$ (and therefore also $\left.\mathbf{q}^{B}(\alpha, \boldsymbol{\omega})=\mathbf{q}^{B}\left(\alpha^{\prime}, \boldsymbol{\omega}\right)\right)$. Then the first order conditions 
for welfare maximization program give

$$
\alpha=\frac{\frac{\partial u^{B}\left(\mathbf{q}^{B}(\alpha, \boldsymbol{\omega})\right)}{\partial q_{i}^{B}}}{\frac{\partial u^{A}\left(\mathbf{q}^{A}(\alpha, \boldsymbol{\omega})\right)}{\partial q_{i}^{A}}}=\frac{\frac{\partial u^{B}\left(\mathbf{q}^{B}\left(\alpha^{\prime}, \boldsymbol{\omega}\right)\right)}{\partial q_{i}^{B}}}{\frac{\partial u^{A}\left(\mathbf{q}^{A}\left(\alpha^{\prime}, \boldsymbol{\omega}\right)\right)}{\partial q_{i}^{A}}}=\alpha^{\prime} .
$$

This contradiction proves the lemma.

Lemma 2. If the solution to the welfare maximization program is interior, then $W(\alpha, \boldsymbol{\omega})$ is strictly convex in $\alpha$.

Proof. Consider two values $\alpha, \alpha^{\prime}, \alpha \neq \alpha^{\prime}$ and let

$$
\alpha^{\prime \prime}=\gamma \alpha+(1-\gamma) \alpha^{\prime}
$$

for some $\gamma \in[0,1]$. Let $\mathbf{q}^{A}, \mathbf{q}^{B}, \mathbf{q}^{\prime A}, \mathbf{q}^{B}$ and $\mathbf{q}^{\prime \prime A}, \mathbf{q}^{\prime \prime B}$ be the optimal values for the maximization problem with $\alpha, \alpha^{\prime}$ and $\alpha^{\prime \prime}$ respectively and total endowment $\boldsymbol{\omega}$. Then,

$$
\begin{aligned}
\gamma W(\alpha, \boldsymbol{\omega})+(1-\gamma) W\left(\alpha^{\prime}, \boldsymbol{\omega}\right)= & \gamma \alpha u^{A}\left(\mathbf{q}^{A}\right)+\gamma u^{B}\left(\mathbf{q}^{B}\right) \\
& +(1-\gamma) \alpha^{\prime} u^{A}\left(\mathbf{q}^{\prime A}\right)+(1-\gamma) u^{B}\left(\mathbf{q}^{\prime B}\right) \\
\geq & \gamma \alpha u^{A}\left(\mathbf{q}^{\prime \prime}\right)+\gamma u^{B}\left(\mathbf{q}^{\prime \prime B}\right) \\
& +(1-\gamma) \alpha^{\prime} u^{A}\left(\mathbf{q}^{\prime \prime}\right)+(1-\gamma) u^{B}\left(\mathbf{q}^{\prime \prime B}\right) \\
& =\alpha^{\prime \prime} u^{A}\left(\mathbf{q}^{\prime \prime}\right)+u^{B}\left(\mathbf{q}^{\prime \prime}\right)=W\left(\alpha^{\prime \prime}, \boldsymbol{\omega}\right) .
\end{aligned}
$$

The inequality follows from the fact that $\mathbf{q}^{A}, \mathbf{q}^{B}$ (resp. $\mathbf{q}^{\prime A}, \mathbf{q}^{B}$ ) solve the maximization problem for $\alpha$ (resp. $\alpha^{\prime}$ ) and that $\mathbf{q}^{\prime \prime}, \mathbf{q}^{\prime \prime B}$ satisfy the feasibility constraint $\mathbf{q}^{\prime \prime A}+\mathbf{q}^{\prime \prime B}=\boldsymbol{\omega}$. Intuitively, the result follows from the fact that the objective function takes the maximum of a set of linear equations. then

Using Lemma 1, we also have that, if $\gamma \in] 0,1\left[, \mathbf{q}^{A} \neq \mathbf{q}^{\prime \prime}\right.$ and $\mathbf{q}^{B} \neq \mathbf{q}^{\prime \prime B}$,

$$
\alpha u^{A}\left(\mathbf{q}^{A}\right)+u^{B}\left(\mathbf{q}^{B}\right)>\alpha u^{A}\left(\mathbf{q}^{\prime \prime A}\right)+u^{B}\left(\mathbf{q}^{\prime \prime B}\right) .
$$

If not, we would have that

$$
\alpha u^{A}\left(\mathbf{q}^{A}\right)+u^{B}\left(\mathbf{q}^{B}\right)=\alpha u^{A}\left(\mathbf{q}^{\prime \prime A}\right)+u^{B}\left(\mathbf{q}^{\prime \prime B}\right) .
$$

By strict concavity, any convex combination of the bundles $\mathbf{q}^{A}$ and $\mathbf{q}^{\prime \prime}$ and the same convex combination of $\mathbf{q}^{B}$ and $\mathbf{q}^{\prime \prime B}$ would also be feasible and provide higher utility to both $A$ and $B$. However, this contradicts the optimality of $\mathbf{q}^{A}$ and $\mathbf{q}^{B}$. As such, the weak inequality for the convexity proof can be replaced by a strict inequality (if $\gamma \in] 0,1[$ ) which shows that $W(\alpha, \boldsymbol{\omega}$ ) is strictly convex in $\alpha$. 


\section{References}

Balasko, Y., 1975. Some results on uniqueness and on stability of equilibrium in general equilibrium theory. Journal of Mathematical Economics.

Balasko, Y., 1978. The transfer problem and the theory of regular economies. International Economic Review 44, 167-179.

Balasko, Y., 1992. The set of regular equilibria. Journal of Economic Theory $58,1-8$

Balasko, Y., 2014. The transfer problem: A complete characterization. Theoretical Economics 9, 435-444.

Bhagwati, J., Brecher, R., Hatta, T., 1983. The generalized theory of transfers and welfare: Bilateral transfers in a multilateral world. American Economic Review 73, 606-618.

Chichilnisky, 1983. The transfer problem with three agents once again. Journal of Development Economics 13, 237-248.

Chichilnisky, G., 1980. Basic goods, the effects of commodity transfers and the international economic order. Journal of Development Economics 7, 505-519.

Dixit, A., 1983. The multi-country transfer problem. Economics Letters 13, $49-53$.

Gale, D., 1974. Exchange equilibrium and coalitions. Journal of Mathematical Economics 1, 63-66.

Geanakoplos, Heal, 1983. A geometric explanation of the transfer paradox in a stable economy. Journal of Development Economics 13, 223-236.

Ginsburgh, V., Waelbroeck, J., 1979. A note on the simultaneous stability of tâtonnement processes for computing equilibria. International Economic Review 20, 367-380.

Leontief, W., 1947. Introduction to a theory of the internal structure of functional relationships. Econometrica 15, 361-373. 
Majumdar, M., Mitra, T., 1985. A result on the transfer problem in international trade theory. Journal of International Economics 19, 161-170.

Mantel, R. R., 1971. The welfare adjustment process: Its stability properties. International Economic Review 3, 415-430.

Mundell, R. A., 1960. The pure theory of international trade. The American Economic Review 50, 67-110.

Negishi, T., 1960. Welfare economics and existence of an equilibrium for a competitive economy. Metroeconomica 12, 92-97.

Polemarchakis, 1983. On the transfer paradox. International Economic Review 24, 749-760.

Postlewaite, A., Webb, W., 1984. The possibility of recipient harming, donor benbenefit transfers with more than two countries. Journal of International Economics 16, 357-364.

Safra, Z., 1984. On the frequency of the transfer paradox. Economics Letters 15, 209-212.

Samuelson, P. A., 1952. The transfer problem and transport costs: The terms of trade when impediments are absent. Economic Journal 62, 278-304.

Samuelson, P. A., 1954. The pure theory of public expenditure. The Review of Economics and Statistics 36, 387-389.

Srinivasan, Bhagwati, 1983. On the transfer paradox and immiserizing growth. Journal of Development Economics 13, 217-222.

Yano, M., 1983. Welfare aspects of the transfer paradox. Journal of International Economics 15, 271-289. 